INPLASY

PROTOCOL

To cite: Silva et al. Front crawl swimming coordination: a systematic review. Inplasy protocol 202140094. doi: 10.37766/inplasy2021.4.0094

Received: 18 April 2021

Published: 18 April 2021

Corresponding author:

Ana Flipa Silva

anafilsilva@gmail.com

Author Affiliation:

Instituto Politécnico da Maia

Support: SFRH/BD/

87780/2012.

Review Stage at time of this submission: Data analysis.

Conflicts of interest:

None declared.

\section{Front crawl swimming coordination: a systematic review}

Silva, AF1; Seifert, L2; Fernandes, R33; Vilas-Boas, JP4; Figueiredo, $\mathrm{P}^{5}$.

Review question / Objective: The aim was to understand the effects of biomechanical, physiological, and personal (gender, skill level, and age) factors on coordination and performance. Condition being studied: Several constraints that are environmental (e.g. aquatic resistance, temperature and viscosity), organismic (e.g. anthropometry, buoyancy, and others globally linked to age, gender, skill level, swim specialty, breathing preference and motor laterality) and taskrelated (e.g. imposed swim speed or stroke rate, use of paddle and fins) impact motor coordination and swimming performance. As motor coordination requires structurally organising intra- and inter-limb coupling, the purpose of this review was to update the state of the art concerning coordination between the upper-limbs in front crawl swimming.

INPLASY registration number: This protocol was registered with the International Platform of Registered Systematic Review and Meta-Analysis Protocols (INPLASY) on 18 April 2021 and was last updated on 18 April 2021 (registration number INPLASY202140094).

\section{INTRODUCTION}

Review question / Objective: The aim was to understand the effects of biomechanical, physiological, and personal (gender, skill level, and age) factors on coordination and performance.

Condition being studied: Several constraints that are environmental (e.g. aquatic resistance, temperature and viscosity), organismic (e.g. anthropometry, buoyancy, and others globally linked to age, gender, skill level, swim specialty, breathing preference and motor laterality) and task-related (e.g. imposed swim speed or stroke rate, use of paddle and fins) impact motor coordination and swimming performance. As motor coordination requires structurally organising intra- and 
inter-limb coupling, the purpose of this review was to update the state of the art concerning coordination between the upper-limbs in front crawl swimming.

\section{METHODS}

Participant or population: Swimmers or triathletes, evaluated in front crawl swimming, with all ages from any sex or skill, without injury, illness or other clinical condition.

Intervention: Front crawl swimming coordination.

Comparator: Skill level, gender and swimming distance.

Study designs to be included: Randomized and non-randomized controlled and/or parallel trials, with no significant differences between groups in baseline assessment of the main outcome.

Eligibility criteria: It was included swimmers or triathletes, evaluated in front crawl swimming, with all ages from any sex or skill, without injury, illness or other clinical condition. It were excluded animal studies, disabled swimmers, other sport modality, other swimming tecnhique, or swimmers in rehabilitation or in return-toplay programs. The main analysis was front crawl swimming coordination, excluding swiming coordination analysed in other swimming techniques and dry land coordination. As comparator skill level, gender and swimming distance, were considered, excluding other swimming techniques and dry land coordination. The main outcome was swimming coordination patterns, excluding outcomes not related to coordination in front crawl behavior; no information (e.g., mean; standard deviation). Only peer reviewed, original, full-text studies written in English were incuded, excluding reviews, letters to editors, trial registrations, proposals for protocols, editorials.

Information sources: An electronic search was conducted during August 2020, including all relevant files from 1980 until that date, using a combination of two keywords ("swim"” and "coordina*"). The search was conducted using bibliographic databases of peer-reviewed literature for sports and sports medicine research: SPORTDiscus, CINAHL, MEDLINE, Scopus, Science Direct, Academic One File, ISI Web of Science, PubMed.

Main outcome(s): Swimming coordination patterns.

Quality assessment / Risk of bias analysis: For the quality assessment/ risk of bias analysis, it was used the methodological index for non-randomized studies (minors).

Strategy of data synthesis: The data synthesis was divided in the different parameters that influences in front crawl swimming coordination divided in the different main groups of parameters: i) biomechanics; ii) energetics; and iii) gender, skill level and maturation.

Subgroup analysis: The analysis was divided in three subgroups: i) How do biomechanical variables interact with swimming coordination?; ii) How do physiological variables influence swimming coordination?; and iii) Do gender, skill level and maturation stage limit the coordination mode?

Sensitivity analysis: Nothing to declare.

\section{Language: English.}

Country(ies) involved: Portugal and France.

Keywords: swimming; coordination; biomechanics; energy cost; expertise.

Contributions of each author:

Author 1 - Ana Filipa Silva - The author worked in the methodology, in the the writing the original draft and reviewing and editing the manuscript, and also in the founding acquisition.

Email: anafilsilva@gmail.com

Author 2 - Ludovic Seifert - The author helped in the conceptualization, the methodology, and in the writing the original 
draft and reviewing and editing the manuscript.

Email: ludovic.seifert@univ-rouen.fr

Author 3 - Ricardo Fernandes - The author reviewed and supervised the process and contributed to the founding acquisition.

Email: ricfer@fade.up.pt

Author 4 - João Paulo Vilas-Boas - The author supervised all the process.

Email: jpvb@fadeup.pt

Author 5 - Pedro Figueiredo - The author helped in the conceptualization, the methodology, revision of the manuscript and in the founding acquisition.

Email: pedrofigueiredo@ismai.pt 